\title{
Study on Absolute Positioning Technique for Medium-Low Speed Maglev Train Based on Cross Coding Inductive Loop Wire
}

\author{
Wei $\mathrm{Nai}^{1,2}$ a, Xiang $\mathrm{Li}^{3,2}$ b, $\mathrm{Yi} \mathrm{Yu}^{2, \mathrm{c}}$, Shaoyin Wang ${ }^{4, \mathrm{~d}}$ \\ ${ }^{1}$ Postdoctoral Research Station of Control Science and Engineering, Tongji University, Shanghai, \\ 201804, China \\ ${ }^{2}$ The Cooperative Center for Maglev and Rail Transit Operation Control System, Tongji University, \\ Shanghai, 201804, China \\ ${ }^{3}$ Key Laboratory of Road and Traffic Engineering, Ministry of Education, Tongji University, Shanghai, \\ 201804, China \\ ${ }^{4}$ Tongji Zhejiang College, Jiaxing, 314051, China \\ aemail: alexni@tongji.edu.cn, ${ }^{\mathrm{b} e m a i l: ~ 1250464591 @ q q . c o m}{ }^{\mathrm{c}}$ email: 12164@tongji.edu.cn, ${ }^{\mathrm{d}}$ email: \\ 82730@tongji.edu.cn
}

Keywords: Medium-Low Speed Maglev; Absolute Positioning; Positioning Accuracy; Cross Coding Inductive Loop Wire

\begin{abstract}
During recent years, the medium-low speed maglev system brings new chances for the development of maglev train related techniques, and traditional operation and control standards have been applied in its signal system design. However, as there are no wheel sets on maglev train, the positioning function cannot be realized by the corresponding method in wheel-rail based urban rail transit. Many related research has already proved that the absolute positioning can be realized by parallel arranged cross coding inductive loop wires with different cross cycles itself without transponders; however, the performance and the precision of positioning for maglev system by using such positioning method still requires to be demonstrated. In this paper, based on a thorough study on cross inductive loop wire which have been applied in different areas, a system design for medium-low speed maglev train positioning based on cross coding inductive loop wire has been proposed, and the arrangement of address line has also been optimized. Performance analysis has shown that the feasibility of the system design and has proved that the optimization of radio arrangement can double the accuracy in train positioning.
\end{abstract}

\section{Introduction}

The medium-low speed maglev system which has been recognized as urban rail transit oriented way of transportation has brought new chances for the development of maglev train related techniques, for several major cities like Changsha and Beijing have already started their construction project of medium-low speed maglev since the year of 2014, and the medium-low speed maglev system related standard has also been in acceleration of formation and improvement [1]. The non-touching design of medium-low speed maglev train and the track, as well as the specific traction and breaking method for the train, have brought distinct differences between maglev train and wheel-rail based train, and have also brought essential difference in realizing key function such as positioning between its operation and control system and wheel-rail based system. The traditional mechanical equipments used in wheel-rail based train for absolute positioning like accelerometer cannot work anymore in maglev trains, therefore, suitable method for absolute positioning for maglev train must be found to make the operation and control a sound system.

As it comes to solve the positioning problem of maglev train, J. Wu et al. (2011) have pointed out that for high speed maglev train, the information of absolute position point can be get from the positioning marking plate, and the relative position information can be read by counting the slot number of alternating current (AC) wire, for the civil feature of high speed maglev track is fixed [2]. However, as the excitation coil is set up on the train itself, the civil structure has no fixed feature for 
medium-low speed maglev system, thus, the absolute positioning for medium-low speed maglev train should not depend on the civil structure. Z. Li (2006) has demonstrated the advantages of realizing position and velocity measuring for maglev train by employing cross inductive loop wire and transponders, and has pointed out that the cross inductive loop wire which has periodical cross point can not only realize effective positioning, but can eliminate the interference generated by spatial long wave radio and its low frequency high order harmonic [3]. Actually, cross coding inductive loop wire which has a bunch of parallel arranged wires with different cross cycles has a wide range of application in automation industries like smelting and warehouse. W. Cheng et al. (2011) and J. Chen et al. (2011) have built a cross coding inductive loop wire based information system, and have pointed out that the cross coding inductive loop wire can realize high resolution positioning in industries, and the resolution can even reach $2 \mathrm{~mm}$ accuracy [4-5]; $\mathrm{H}$. Li and C. Nie (2007) have designed a system applied in coke oven operation site, and have pointed out that the positioning based on cross coding inductive loop wire can meet the accuracy requirement in such industry [6], whereafter, W. Cheng et al. (2010) has done specific study on the coding issue, and has pointed out that the Gray Code has better positioning performance [7]; W. Bie and X. Miao (2009) have made a study on warehouse positioning, and have found that the cross coding inductive loop wire works well in positioning matrix in finding target storage rack [8]; while J. Chen and X. Yang (2014) have studied the interference in the signal receiving process of the positioning system based on cross coding inductive loop wire, and have proposed a scheme in reducing such interference [9].

It cannot be so hard to find out that, even if there are some related research in cross coding inductive loop wire, studies on its application in maglev train system are still hard to find; but fortunately, many related research has already proved that the parallel arranged cross coding inductive loop wires with different cross cycles can realize absolute positioning just by itself and has high positioning accuracy. As there are not so much difference in basic ideas between those automation industries and the maglev system, the only thing which have to be demonstrated is the performance and the precision of positioning for maglev system by using cross coding inductive loop wire. In this paper, based on a thorough study on cross coding inductive loop wires which have been applied in different areas, a system design for medium-low speed maglev train positioning based on cross coding inductive loop wire has been proposed, and the arrangement of address line has also been optimized. Performance analysis has shown that the feasibility of the system design and has proved that the optimization of radio arrangement can double the accuracy in train positioning.

\section{Basic Concepts for Train Positioning}

In wheel-rail based urban rail transit system, the traction energy is forced onto the wheel-rail gears so as to realize train running after it has been converted into mechanical energy. In early stage, as the train speed is not quite high, only track circuit formed by two steel tracks and the wheel sets which are made of metal material has been utilized to determine whether a section of rail has been occupied. Then, as the train speed has ever been in an increasing process, and the performance requirements of automatic train control has ever been in an improving process, the operation control system (OCS) has to collect quasi real-time positioning information so as to enhance its function. In this context, the accelerometer, which can convert the mechanical energy of wheel rotation into the velocity parameter, has been widely used in wheel-rail positioning system.

Accelerometer combined with transponder, can calculate that how much distance has been passed after a certain transponder, and can acquire the absolute position of the train in the track. However, idling (wheel rotates without train moving) and slipping (train moves without wheel rotation) are unavoidable drawbacks which will bring accumulated error in positioning. In order to eliminating such error, radar is also used as a auxiliary way in acquiring the relative train position apart from the accelerometer.

Consider that in medium-low speed maglev train system, the non-touching design has been realized between the train and the rail track, the accelerometer applied in wheel-rail system cannot work anymore, alternative positioning method must be employed. Thus, there is an essential 
difference in relative as well as absolute positioning between medium-low speed maglev train system and wheel-rail system. According to the thorough study on cross inductive loop wires in the literature review part of this paper, it can be seen that the cross inductive loop wire is an effective positioning method for moving objects, its working principle of relative positioning can be shown in Figure 1.

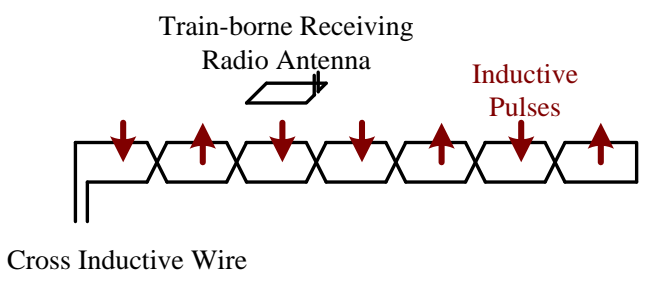

Fig.1. Work principle of cross inductive loop wire in acquiring relative position for medium-low speed maglev train

There are cross points along the rail track every fixed distance for the cross inductive loop wire, and the current direction of the inductive wire loop in such fixed distance after charging is opposite with the neighbor loops; as there is a corresponding train-borne receiving loop-shaped radio antenna which detect the position of the medium-low speed maglev train by employing electromagnetic induction principle, the inductive electromotive force will return to 0 when the center of antenna overlaps with the cross points of the inductive wire, and the inductive electromotive force will reach its highest value when the center of antenna overlaps with the center of each loop. Therefore, as the medium-low speed maglev train goes through the cross inductive loop wire distributed along the track, periodical changing inductive electromotive force can be generated. The relative position can thus be acquired by counting the changing times, and the absolute position can also be acquired by combined the relative position information with the coordinate of the transponders.

\section{Positioning System Design for Medium-Low Speed Maglev}

Cross coding inductive loop wire is an absolute positioning equipment for non-touching moving objects based on inductive address code. The address code is formed according to the design of each cross inductive loop wire parallel arranged together. Each cross inductive loop wire has different length between cross points, and represents different coding bits of the whole coding inductive loop wire. The basic working principle of cross coding inductive loop wire is shown in Figure 2.

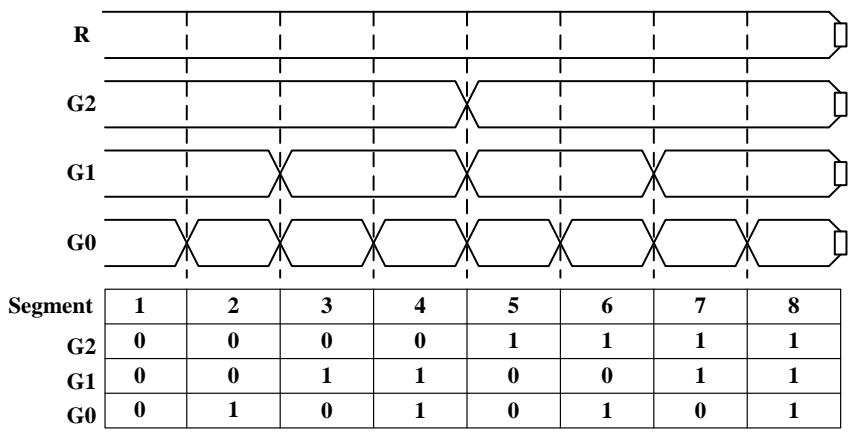

Fig.2. Work principle of cross coding inductive loop wire in acquiring absolute position for medium-low speed maglev train

Among all the cross inductive loop wires set up on the track, one of them is serving as the reference line (marked with R), while the others are serving as the address lines (marked with G0, G1, G2, ...), and the combination coding describes the absolute position of the train-borne antenna. Each cross inductive loop wire for coding has the same working principle as the regular cross inductive loop wire. Setting the corresponding number of time slots with the address lines, the code of each address line can be acquired in each time slot by comparing the inductive electromotive force generated by each address line and the one generated by the reference line. If the signal from reference line is set as the standard signal " 0 ", then the inductive pulse in the same direction with the reference signal can be coded as " 0 ", and the inductive pulse in the opposite direction with the 
reference signal can then be coded as " 1 ”. Figure 2 shows an cross coding inductive loop wire positioning system with only 3 address lines, therefore, in a time period with 3 time slots, a 3-bit address code can be acquired, and this address code reflects the absolute position of the maglev train.

It can be seen that the basic working principle of cross coding inductive loop wire is based on cross inductive loop wire, and it is a kind of combined application which expands the function of cross inductive loop wire. As different address code represents different position area of the track, the absolute positioning method based on cross coding inductive loop wire does not have to reference the information from transponders.

By comprehensively consider the positioning accuracy and the extension of positioning distance, in the application of cross coding inductive loop wire, a 11-bit address coding scheme has been used in the $1.7 \mathrm{~km}$-long experimental line in Shanghai Lingang New City, namely, the cross coding inductive loop wire based absolute positioning equipment has 12 wire lines including 1 reference line and 11 address lines in it. The minimum length of the between cross points in the lowest address line is $10 \mathrm{~cm}$, which means the precision in this experimental line can reach a positioning accuracy up to $10 \mathrm{~cm}$. The extension of positioning distance is $102.4 \mathrm{~m}$, which means the 11-bit address code will repeat every $102.4 \mathrm{~m}$, therefore, the whole experimental line must be divided into several 102.4-meter-long sections and each section should be distributed a sole section number. In the main track, switch and the train garage of the experimental line, 19 sections can be distributed as shown in Figure 3.

As discussed above, each positioning section of cross coding inductive loop wire only has a length at $102.4 \mathrm{~m}$, the track length of medium-low speed maglev is generally way much longer than that, therefore, ground stations has been set along the line and each station covers 4 positioning sections with a total length of $409.6 \mathrm{~m}$. Thus, the whole line can be connected by $\mathrm{n}$ ground stations, and there are no physical connection between stations, the only way to identify the station is realized by station code. Train-borne positioning module can process the station code and address code via dedicated positioning software, by this mean, address code in neighbor stations can be connected even if there are no physical connection between them, and the absolute position information can be acquired. Both the station code and the address code are presented by binary bit units, and it can be easily seen that a 3-bit-long station code can describe 8 different ground stations. To be specific, for the $1.7 \mathrm{~km}$ experimental line in Shanghai Lingang New City, 6 ground stations are set as shown in Figure 3.

It can be easily found from section III-A that the positioning accuracy of cross coding inductive loop wire depends on the very address line which has the shortest cycle of cross points. Back to the example shown in Figure 2, G0 is the address line which has the shortest cycle of cross points and divided the section into 8 segments, as the maglev train passes such positioning section, address line G2, G1 and G0 will form a 3-bit address code and will be detected by the train-borne antenna so as to let the on-board system get the address information. Suppose that the cycle of cross points of line $\mathrm{G} 0$ is $2 \mathrm{~m}$, the positioning accuracy of this 3-bit address code would just be $2 \mathrm{~m}$ according to Figure 2. However, as the arrangement of each cross inductive wire can be different from the way shown in Figure 2, the absolute positioning realized by cross coding inductive loop wire might be optimized by changing the way of address line arrangement. Here is this section, an optimized way of address line arrangement scheme has been proposed, by comparing with the example shown in Figure 2, the optimized scheme is shown in Figure 4.

From Figure 4, it can be seen that the arrangement of address line G0 has been changed comparing with the original way, moving the address line G0 by half of its cross cycle ahead, can on some level improve the positioning accuracy of the 3-bit-long address code, for the span represented by each 3-bit-long address code has been changed from a whole cross cycle of address line G0, to just half of it. In other words, if the cross cycle of address line G0 is still $2 \mathrm{~m}$, the positioning accuracy of the optimized cross coding inductive loop wire can reach up to $1 \mathrm{~m}$, which means that the positioning accuracy of the cross coding inductive loop wire is significantly improved without even changing the hardware configuration of it. 


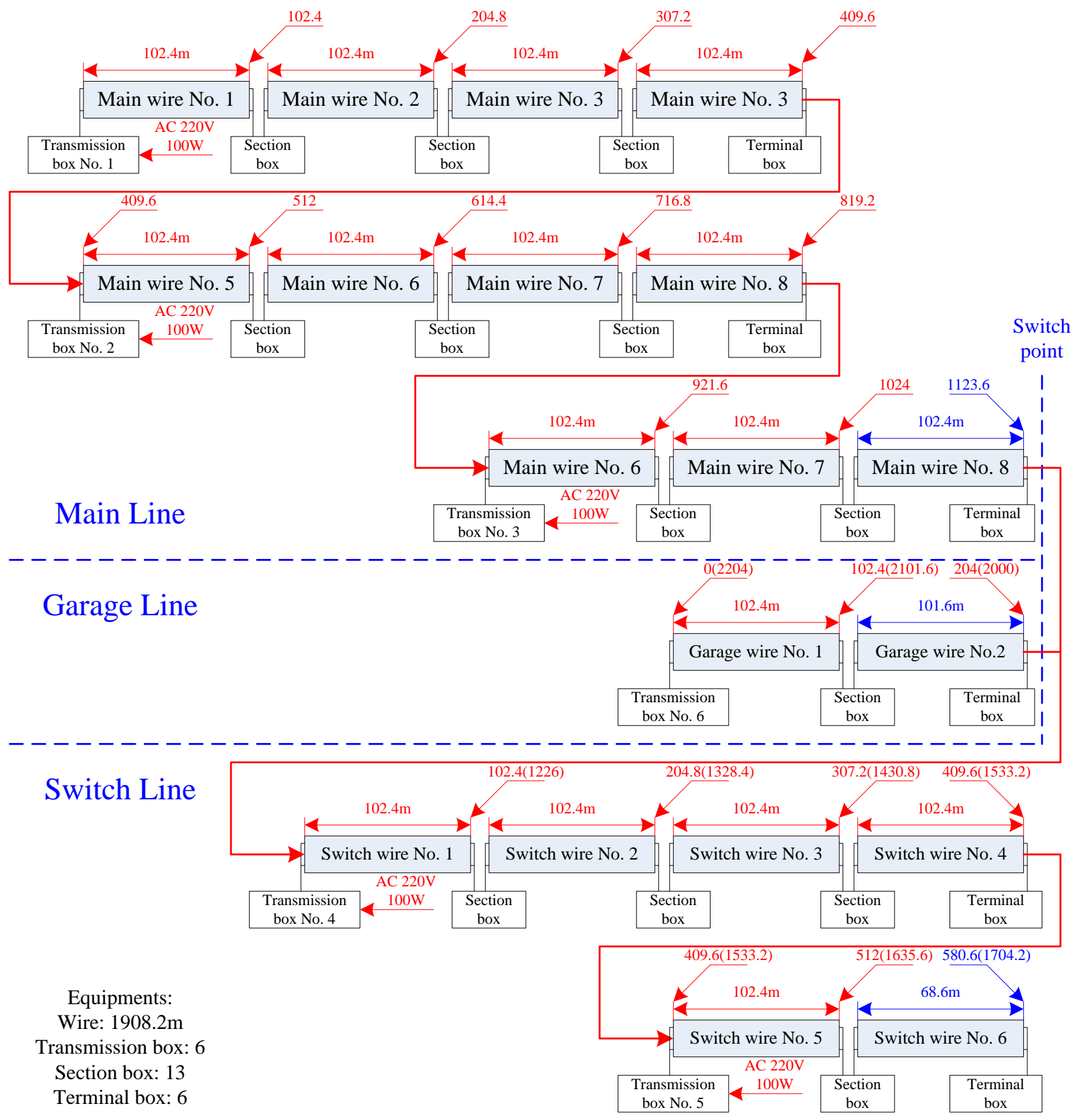

Fig.3. Address coding scheme for $1.7 \mathrm{~km}$ experimental line in Lingang New City

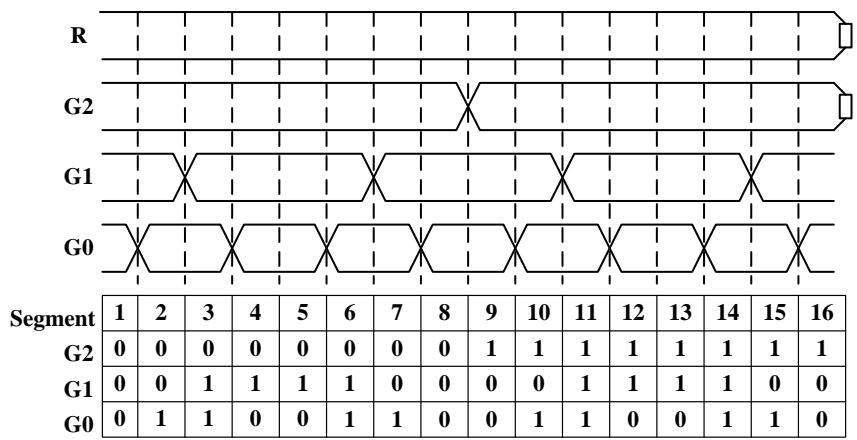

Fig.4. Work principle of cross coding inductive loop wire in acquiring absolute position after optimizing the arrangement of address lines

As it comes to the performance of such optimized cross coding inductive loop wire, it should be firstly pointed out that there are repeats of address code in each section, for example, code 001 appears twice in both segment 2 and segment 7, therefore, certain algorithm should be applied to read present address code as well as the last address code so as to determine which segment the 
antenna is located in. It would be worthwhile for the positioning system to improve the accuracy by just read a several-bit-long address code. Apart from the address code repeat issue, it can be found from Fig. 4 that, as the arrangement of address line G0, the 3-bit-long address code in this scheme is either the same with its neighbor code, or just different in only 1 bit. According to the result analysis in literature [7], the influence for the optimized positioning system will be less if any bit error happens.

\section{Conclusion}

In this paper, thorough studies on train positioning method based on cross inductive wire and cross coding inductive loop wire have been done, the applicability of cross coding inductive loop wire in absolute positioning for medium-low speed maglev has been analyzed, and the corresponding system design has been done. Moreover, an optimized arrangement method for address lines in cross coding inductive loop wire has been proposed, which put the lowest address line half cross cycle ahead; and the positioning accuracy can be doubled by employing it. The results acquired in this paper would be meaningful for the positioning system based on cross coding inductive loop wire, and would serve as a theory basis of system design in the operation and control system of medium-low speed maglev train.

\section{Acknowledgement}

This work is supported by National Key Technology R\&D Program of the 12th Five-year plan, Systematic Study on Engineering Integration of High Speed Maglev Transportation, Grant No. 2013BAG19B01, and Fund of Shanghai Cooperative Center for Maglev and Rail Transit Operation Control System.

\section{References}

[1] Ministry of Housing and Urban-Rural Construction of the People's Republic of China. Technical Standards of Operation and Control System for Medium-Low Speed Maglev Transportation [S], 2015.

[2] J. Wu, W. Zhou, L. Li. Research on speed and position detection system of high speed maglev train [J], Journal of National University of Defense Technology, 2011 33(1) 109-114.

[3] Z. Li. Design and Realization of Communication and Positioning and Velocity Measuring System for Maglev Train Based on New Looped Cable [D], Master's thesis, Changsha: National University of Defense Technology, 2006.

[4] W. Cheng, J. Chen, X. Chen. research on position detection system with high resolution based on inductive radio [J], Process Automation Instrumentation, 2011 32(6) 32-35.

[5] J. Chen, X. Chen, W. Chen, Method of induction radio based position detection at vehicle station [J], Journal of Electronic Measurement and Instrument, 2011 25(3) 272-278.

[6] H. Li, C. Nie. Coke oven locomotive interlocking and automotive running control system [J], Journal of Hunan Institute of Science and Technology (Natural Sciences), 2007 20(4) 63-68.

[7] W. Cheng, J. Chen, X. Chen, Research on induction radio technology and its application in position detection [J], Journal of Hunan Institute of Science and Technology (Natural Sciences), 2010 23(4) 62-65.

[8] W. Bie, X. Miao, Coding cable-based address detection in automated steric warehouse [J], Journal of Lanzhou University of Technology, 2009 35(2) 88-92.

[9] J. Chen, X. Yang, Method of improving position detection resolution by induction radio [J], Journal of Electronic Measurement and Instrument, 2014 28(9) 943-950. 